REVIEW

\title{
Isolated mild fetal ventriculomegaly
}

\author{
M Wyldes, M Watkinson
}

Arch Dis Child Fetal Neonatal Ed 2004;89:F9-F13

Ventriculomegaly is an excess of fluid in the lateral ventricles within the developing cerebrum. It is usually diagnosed at a routine fetal anomaly scan at 18-22 weeks gestation. Management of the condition and counselling of parents are difficult, as the cause, absolute risk, and degree of resulting handicap cannot be determined with confidence.

See end of article for authors' affiliations .....................

Correspondence to: Dr Watkinson; michael.watkinson@ heartsol.wmids.nhs.uk

Accepted 8 December 2002

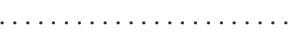

$\mathrm{V}$ entriculomegaly is an excess of fluid in the lateral ventricles within the developing cerebrum. The diagnosis is most often identified at a routine fetal anomaly scan, traditionally undertaken at 18-22 weeks gestation. Earlier in pregnancy the appearances of the fetal cerebrum are dramatically different, with the fluid filled ventricles occupying most of the available space. The appearances change rapidly from 16 weeks onwards, such that there is more brain tissue and less fluid. Ventriculomegaly is defined as an axial diameter $>10 \mathrm{~mm}$ across the atrium of the posterior or anterior horn of lateral ventricles at any gestation ${ }^{1}$ (fig 1). The atrial diameter is constant at $7.6(0.6) \mathrm{mm}$ (mean (SD)) from 14 to 38 weeks gestation. ${ }^{1}$ A separation of more than $3 \mathrm{~mm}$ of the choroid plexus from the medial wall of the lateral ventricle is also used to define the abnormality ${ }^{2}$ (fig 2). The separation of the choroid plexus is often greater than 3 or even $4 \mathrm{~mm}$ even when the ventricular measurement is borderline, and this can be a useful measurement to follow over time. The term hydrocephalus is used for fetal ventricles with rapidly, and is not considered further here.

The original criteria for enlargement of the cerebral ventricles were based around the measurement of the lateral border of both the anterior and posterior horns from the midline, in comparison with the largest diameter of the hemisphere. ${ }^{3}$ This ventriculo-hemispheric ratio was used for many years because the image available for assessment often contained only the outline of the skull, the cavum of the septum pellucidum, and the reflection of the lateral borders of the anterior and posterior horns. With increasing image quality, it is now possible to see the medial border of the ventricles, and therefore to assess the ventricle itself directly.

The fetal biparietal diameter at 20 weeks gestation measures about $48 \mathrm{~mm}$ in diameter, and, depending on the size and shape of the mother, may lie up to $20 \mathrm{~cm}$ away from the ultrasound probe. Therefore the image quality obtained in many cases is suboptimal, especially compared with the neonatal head scan, where an atrial diameter $>15 \mathrm{~mm}$ and often enlarging high frequency probes can be applied directly to the fontanelles. The currently recommended checklist for anomaly scanning at 20 weeks includes clear views of the cerebral ventricles.

\section{ROLE OF FETAL MEDICINE}

The assessment of the fetal head includes an assessment of the shape of the skull, which should be smoothly ovoid. The biparietal diameter and head circumference are measured at the level of the cavum of the septum pellucidum. At this level, and slightly superior in the same plane, the lateral ventricles are seen. Modern imaging technology allows the choroid plexus to be clearly seen as a dumb-bell shaped echogenic structure occupying the central portion of the lateral ventricle. Both anteriorly and posteriorly, the cerebrospinal fluid can be seen, and a measurement can be made across the anterior and posterior horns.

The diagnosis of fetal ventriculomegaly is being made more often, and specialists in fetal medicine assess and manage cases under the following headings.

(1) Ensure that there are no associated structural abnormalities

\section{A. General structural survey}

a. Central nervous system: neural tube defects; agenesis of the corpus callosum; Dandy-Walker malformation

b. Cardiac anomalies

c. Other structural anomalies

B. Soft markers for trisomy
a. Nuchal pad
b. Echogenic bowel
c. Echogenic focus in the heart
d. Dilated renal pelvis
e. Choroid plexus cyst
f. Short femur/humerus

(2) Discuss possible causes and arrange investigations
A. Trisomy 21
B. Other karyotype anomalies
C. Toxoplasmosis \& CMV

(3) Inform the parents of the problem and the possible future implications
A. What is the risk of any handicap
B. What types of problems are reported

(4) Consideration of termination of pregnancy 


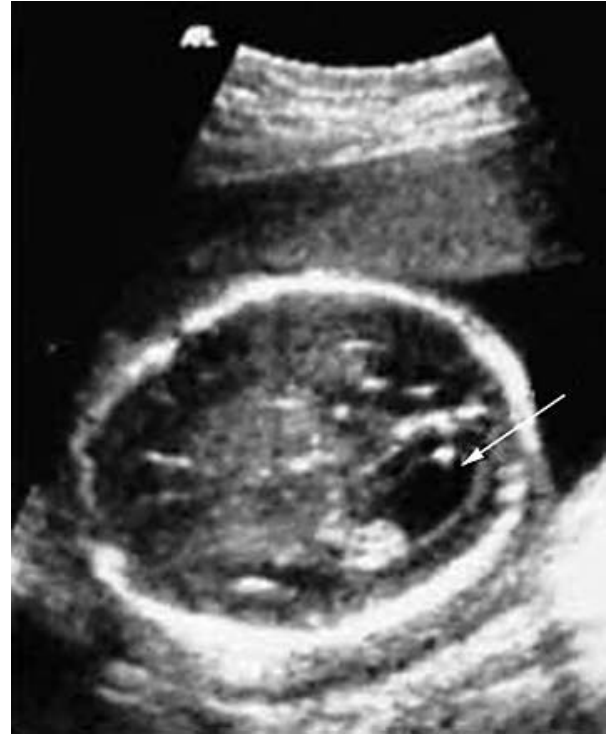

Figure 1 Mild fetal ventriculomegaly $(11 \mathrm{~mm})$ with enlargement of the posterior horn of the lateral ventricle (arrowed).

\section{BREAKING THE NEWS}

Consultations in which bad news is broken are difficult, and need to be tailored to the individual mother or couple. The presence of a neonatologist and an experienced midwife counsellor is important, but even the most experienced teams will often find this element of care the most testing. It should be accepted by the professionals at the outset that the requirements and reactions of different people will vary tremendously. Most will openly show distress, and will accept the findings of "a problem", but some will produce a series of difficult questions as part of a denial response.

It is traditional to discuss abnormal ultrasound findings in a face to face seated consultation, but some couples prefer actually to see the abnormal findings on the screen before the discussion. The option of showing the problem to the woman, or taking images to demonstrate the findings is helpful to give scale and substance to the problem. A seated consultation away from the scan room will follow. It is necessary briefly to run through the complex issues of diagnosis and management, but it should always be accepted that the amount of information that is retained in this first consultation is likely to be very limited.

A further consultation should follow quickly, ideally within a few days, so that important decisions can be taken and results obtained without delay.

\section{MANAGEMENT OPTIONS}

Ventriculomegaly associated with other abnormal findings, or structural malformations such as spina bifida will usually carry a poor prognosis. ${ }^{4}$ Most of the conditions associated with ventriculomegaly are well defined, and clear information can be given to parents about the outcome of the pregnancy. For example, Dandy-Walker malformation has an $80 \%$ risk of developmental delay in survivors by 4 years of age. ${ }^{6}$

Cases of apparently isolated ventriculomegaly present a more difficult group. Karyotyping will identify chromosomal anomalies in $3-4 \%$ of cases, most often trisomy $21 . .^{7}$ In the absence of a chromosomal abnormality or any structural malformation there remains the possibility of both death and handicap.

The option of termination of the pregnancy needs to be considered. Information needs to be gathered as quickly as

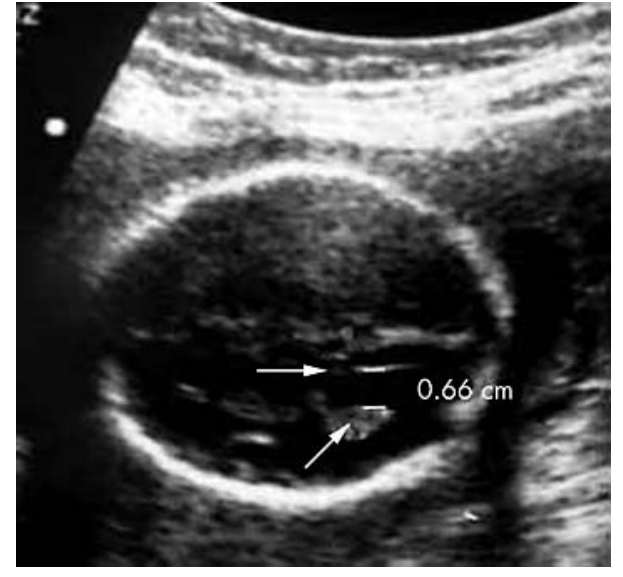

Figure 2 Abnormal "dangle" of the choroid plexus of $0.66 \mathrm{~cm}$, with arrows indicating the medial wall of the ventricle and the choroid plexus.

possible so that the parents are in full possession of the facts. Rapid karyotyping using quantitative fluorescent polymerase chain reaction will give a result within 48 hours of the taking of an amniocentesis sample, ${ }^{8}$ but if parents opt for termination of the pregnancy it may be appropriate to proceed on the basis of the ultrasound findings alone, without waiting for the karyotype. Even in these cases in which termination of pregnancy is arranged, the prenatal karyotype offers the best chance of making the diagnosis of trisomy because karyotyping after termination has a substantial risk of failure of cell culture, $27 \%$ in one series. ${ }^{9}$ Trisomy is important to identify because the management of future pregnancies will be significantly altered by the result. If a woman of any age has had a pregnancy associated with a trisomy, her risk for recurrence is at least $1 \%$, and therefore early karyotyping, usually by chorionic villus sampling, is offered.

Termination of pregnancy with a fetus with stable ventriculomegaly, where the risk of handicap is between $9 \%{ }^{10}$ and $36 \%{ }^{11}$ some of which may be mild, is a difficult and complex issue. In England and Wales, category E of the 1966 Abortion Act states that termination can be undertaken, without limit of gestation, where a significant risk of handicap exists. This is never an easy decision, but as the pregnancy progresses, the decision to terminate becomes harder. Certainly once a pregnancy has advanced past 24 weeks, there are very few women who opt for termination unless the predicted outcome is universally poor.

\section{ANTENATAL PROGRESS}

Once the initial phase of active investigation and a decision on management is taken, the pregnancy is often reviewed up to monthly to determine whether the ventricles return to normal, remain stable, or progress. Some data suggest that the degree of ventriculomegaly, ${ }^{7}{ }^{12}$ and whether it remains stable, progresses, or returns to normal, ${ }^{13}{ }^{14}$ affects the chances of a normal outcome for the child. The prognosis, however, is very variable, and it could be argued that serial ultrasound scanning is unlikely to affect the management. The exceptions are cases of severe ventriculomegaly in which the head circumference is significantly increased. In these cases, a difficult decision needs to be made about the mode of delivery, and an additional ultrasound assessment at the end of pregnancy allows evaluation for labour and delivery.

\section{PLANNING DELIVERY}

If the head circumference is greater than $400 \mathrm{~mm}$, the chances of a vaginal delivery are small. These cases are the very severe end of this spectrum, and poor outcomes are 
usually predicted. However, most cases of isolated mild ventriculomegaly can be delivered vaginally, as the head size will not be significantly increased.

Cases should be discussed with the paediatric team well in advance of delivery, and a definitive plan recorded to be available for the midwifery, obstetric, and neonatal team.

\section{POSTNATAL MANAGEMENT}

The paediatrician has a number of tasks after the birth of a baby with ventriculomegaly. This is true whether the ventriculomegaly was apparently isolated or associated with other abnormalities, and whether it was transient or unilateral or mild.

- Confirming the diagnosis

- Checking for associated abnormalities and excluding other pathologies

- Considering the prognosis of mild ventriculomegaly

- Talking with the parents

- Following the child and screening for disabilities

\section{CONFIRMING THE DIAGNOSIS OF VENTRICULOMEGALY}

Head circumference must be measured accurately and plotted on centile charts. Babies born with isolated mild ventriculomegaly have not suffered raised intracranial pressure in utero, and head circumference is usually within the normal range, and the anterior fontanelle is under normal pressure. Signs contrary to this raise the concern of hydrocephalus. ${ }^{15}$ Ultrasound scans of the brain must be undertaken to look for congenital abnormalities and to measure the ventricles. Neonatal scans are always through the anterior fontanelle, offering a different view from the lateral views obtained antenatally. Standard neonatal measurements of the lateral ventricles do not include the width of the ventricular atrium. The measurement that corresponds most closely to it is Levene's ventricular index. ${ }^{16}$ This is the distance from the falx in the midline to the lateral most point of the ventricle, and is greater than the width of the ventricular atrium. However, as both have established centiles, the centile of the antenatal measurement can be justifiably compared with that taken after birth. Therefore a postnatal ventricular index above Levene's 97th centile (about $14 \mathrm{~mm}$ at term) would support the diagnosis of ventriculomegaly, particularly in the presence of a head circumference below that range. Ventriculomegaly can resolve during gestation, ${ }^{13} 1417$ and the ventricles may appear normal at birth.

\section{CHECKING FOR ASSOCIATED ABNORMALITIES AND EXCLUDING OTHER PATHOLOGY}

Perinatal mortality may be as high as $37 / 1000$ births, often in association with severe intrauterine growth retardation. ${ }^{7}$

The presence of one anomaly mandates a search for others. Many are diagnosed antenatally, but a review of 360 cases of ventriculomegaly from 21 series found that $25 \%$ of babies with apparently isolated fetal ventriculomegaly had additional associated anomalies recognised after birth. ${ }^{14}$ In one series, that figure is as low as $16 \%{ }^{18}$ In another series of 55 fetuses with mild ventriculomegaly, associated anomalies were missed in $37 \%$, but only $2 \%$ of fetuses were misclassified as isolated ventriculomegaly, because at least one anomaly had already been detected in most of those where additional anomalies came to light after birth. ${ }^{19}$ This has important implications for counselling, because of the poorer long term outcome in the presence of other anomalies. Cardiac lesions, oesophageal atresia, and renal, limb, and genital abnormalities have all been reported as "missed" on antenatal scanning. ${ }^{19}$ Associated malformations of the central nervous system including aplasia or hypoplasia of the corpus callosum, Dandy-Walker cyst, lissencephaly, holoprosencephaly, schizencephaly (fig 3), and aqueduct stenosis may be diagnosed for the first time postnatally. Inborn errors of metabolism have also been reported. ${ }^{12}{ }^{13}$ In one United Kingdom series, aneuploidy occurred in 3\% of fetuses with isolated ventriculomegaly, and $36 \%$ of those with additional malformations. ${ }^{18}$ A review of 234 cases found that chromosomal aberrations, most commonly Trisomy 21 , were present in $3.8 \%$ of babies with isolated ventriculomegaly. ${ }^{7}$ Fragile $\mathrm{X}$ should also be considered. ${ }^{20}$

Clinical and serological stigmata of congenital infections must be sought in babies with ventriculomegaly. In Hungary, $47 \%$ of babies with ventriculomegaly (some of whom had ventricles $>15 \mathrm{~mm}$ ) had evidence of congenital infection, with cytomegalovirus and toxoplasmosis accounting for $71 \%$ of these cases. ${ }^{21}$ Some American and European series have reported a low (5\% of fetuses with a $10-15 \mathrm{~mm}$ ventricular atrium $^{12}$ ) or zero incidence ${ }^{22}$ of congenital infection. However, raised fetal serum interferon alpha has been detected significantly more often in a series of French babies with unexplained ventriculomegaly (29\% v1\% in controls). This was suggestive of viral infection, and consequently more extensive viral screening may be indicated. ${ }^{23}$

\section{PROGNOSIS OF ISOLATED MILD VENTRICULOMEGALY}

The paediatrician, in the near certainty of having not missed any associated anomalies, should be able to offer a more precise prognosis than his/her obstetric colleague who may possibly have missed an anatomical anomaly, or may have been refused the opportunity to check the fetal karyotype or metabolism. Yet, considering the wealth of obstetric information on fetal ventriculomegaly, the paediatric data are sparse and of poor quality. Kelly et $a l^{24}$ recently reviewed the literature on the neurological assessment of isolated mild ventriculomegaly. From 15 reported series, they found 337 survivors, but only 288 had been followed, and the reasons for failure to follow the other 49 (15\%) were seldom clear. The lower end of the age range for follow up was less than 12 months in 10 of the 15 series, and in two it was unknown. Yet outcome scores tend to deteriorate during the first 5 years of life, as cognitive developmental problems may be missed when language cannot be accurately assessed. ${ }^{17}$ The assessment test was unknown or unavailable in eight series, and, in another two, a telephone call to a family doctor or an interview was the "test" used for developmental assessment.

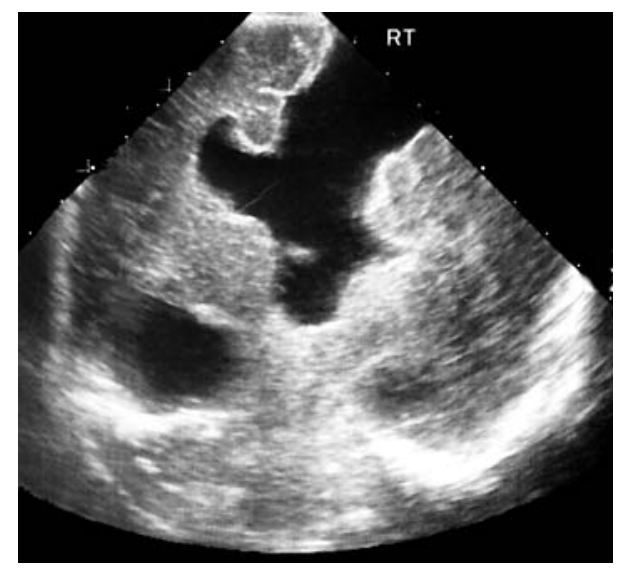

Figure 3 A neonatal coronal ultrasound scan of the head showing right parietal schizencephaly in a baby thought to have isolated ventriculomegaly as a fetus. 
Twining et al ${ }^{4}$ had reviewed this 7 years earlier: of their 10 series of "isolated" fetal ventriculomegaly, only two were in the later review. However, they compared chalk with cheese when juxtaposing a Swiss series ${ }^{25}$ with one from London. ${ }^{18}$ In the former, all 13 babies survived and were shunted, but none had isolated ventriculomegaly, and only three $(23 \%)$ were "normal". In the latter, only 19 of the 58 survived, five were shunted, and $12(63 \%)$ were normal. Overall Kelly et al ${ }^{24}$ reported that $89.6 \%$ of the cases of isolated ventriculomegaly that were followed were normal, and Twining et $a l^{4}$ found $52 \%$ to be normal. Both figures must be treated with great circumspection.

There are two recent series of children confirmed after birth to have had only isolated fetal ventriculomegaly, and in which the developmental assessment test is specified. Bloom et $a l^{11}$ used the Bayley scales of infant development ${ }^{26}$ to assess mental and psychomotor developmental indices in 22 children with ventriculomegaly and 22 matched controls. Eight $(36 \%)$ of the index group but only one of the controls $(\mathrm{p}=0.021)$ were developmentally delayed (score $<85$ on the mental development index) although all had appeared normal at birth. As a group, the children with ventriculomegaly were 8-10 points below the controls in both mental and psychomotor development scores at a mean age of 22 months. Vergani et $a l^{10}$ offered conflicting results. All 45 of their euploid children with isolated mild ventriculomegaly were normal at a median age of 30 months using the MilaniComparetti and Gidoni scale. ${ }^{27}$ However, in reviewing the literature, they suggested that $9 \%$ of affected children were delayed.

Mild non-progressive unilateral ventriculomegaly of 11$13 \mathrm{~mm}$ was regarded by Senat $e t \mathrm{al}^{28}$ as a variation of normal fetal anatomy with a favourable neurological outcome. They reported 14 cases and reviewed another 38: overall 42 (88\%) of the 48 survivors were thought to have normal cognitive development. Three fetuses had been terminated for rapid unilateral ventricular enlargement, and one with multiple malformations was stillborn.

Ventriculomegaly occurs in older people with schizophrenia, but no clear association has been found between fetal ventriculomegaly and psychosis. Nevertheless, some speculate that, as fetal ventriculomegaly can persist through childhood, further understanding of neurodevelopmental disorders may yet emerge and allow recognition of high risk children. ${ }^{29}$

\section{TALKING TO PARENTS}

This confusion of isolated and non-isolated cases, of poor or non-existent standardisation of developmental tests serves the children, their families, obstetricians, and paediatricians poorly. Counselling remains very difficult. As many will be normal, termination of the fetus thought to have mild isolated ventriculomegaly will not have been offered to parents. In general, babies with associated malformations or aneuploidy fare less well than those with truly isolated nonprogressive ventriculomegaly, who probably do slightly less well than those with transient or unilateral ventriculomegaly. The paediatrician must nevertheless advise that isolated nonprogressive ventriculomegaly can only be confidently diagnosed after birth, no matter how skilled his/her obstetric colleagues. Although such children appear normal at birth, as a group they have a 10 point deficit in the mental and psychomotor developmental indices. ${ }^{11}$ Perhaps a fair figure to give to a parent is that 1 in 3 to 1 in 5 children will have developmental delay. Further, their learning, psychological wellbeing, and motor capabilities in school years and beyond have not yet been measured prospectively. There are no data to suggest that children who are normal in the first years of life will have any additional problems as they grow, although one historical series may suggest deteriorating function with age. ${ }^{17}$

\section{FOLLOWING THE CHILD AND SCREENING FOR DISABILITIES}

Children who have had mild isolated fetal ventriculomegaly should be followed until the paediatrician or psychologist is confident that they are functioning within the normal range. Those who do not should be referred to appropriate agencies and carers. Testing should be done with a developmental tool that offers a profile of the different areas of development such as the Bayley scales of infant development ${ }^{26}$ (mental and motor at 2-30 months), the Griffiths scales of mental development $^{30}$ (locomotor, personal social, hearing and speech, eye/hand coordination, and performance at 0-8 years, and practical reasoning above 2 years of age), or the schedule of growing skills ${ }^{31}$ (passive postural, active postural, locomotor, manipulative, visual, hearing and language, interactive social, and self care at $0-5$ years).

\section{CONCLUSION}

Consensus is emerging about the diagnosis and management of fetal ventriculomegaly. However, a persistent problem is the difficulty in counselling parents during pregnancy and after birth, as the cause, absolute risk, and degree of resulting handicap cannot be stated with confidence in many of the affected children.

\section{Authors' affiliations}

M Wyldes, Princess of Wales Maternity Unit, Birmingham Heartlands Hospital, Birmingham, UK

M Watkinson, Neonatal Unit, Birmingham Heartlands Hospital, Birmingham B9 5SS, UK

\section{REFERENCES}

1 Cardoza JD, Goldstein RB, Filly RA. Exclusion of fetal ventriculomegaly with a single measurement: the width of the lateral ventricular atrium. Radiology 1988;169:711-14.

2 Cardoza JD, Filly RA, Podrasky AE. The dangling choroid plexus: a sonographic observation of value in excluding ventriculomegaly. AJR Am J Roentgenol 1988;151:767-70.

3 van Egmond-Linden A, Wladimiroff JW, Niermeijer MF, et al. Fetal hydrocephaly: diagnosis, prognosis and management. Ultrasound Med Biol 1986; 12:939-44

4 Twining $P$, Jaspan $T$, Zuccollo J. The outcome of fetal ventriculomegaly. Br J Radiol 1994;67:23-31.

5 Bromley B, Frigoletto FD, Benacerraf BR. Mild fetal lateral ventriculomegaly: clinical course and outcome. Am J Obstet Gynecol 1991;164:863-7.

6 Aletebi FA, Fung KF. Neurodevelopmental outcome after antenatal diagnosis of posterior fossa abnormalities. J Ultrasound Med 1999:18:683-9.

7 Pilu G, Falco P, Gabrielli S, et al. The clinical significance of fetal isolated borderline ventriculomegaly: report of 31 cases and review of the literature. Ultrasound Obstet Gynecol 1999;14:320-6.

8 Pertl B, Kopp S, Kroisel PM, et al. Quantitative fluorescence polymerase chain reaction for the rapid prenatal detection of common aneuploidies and fetal sex. Am J Obstet Gynecol 1997;177:899-906.

9 Kyle PM, Sepulveda W, Blunt S, et al. High failure rate of post-mortem karyotyping after termination for fetal abnormality. Obstet Gynecol 1996:88:859-62

10 Vergani $\mathbf{P}$, Locatelli A, Strobelt $N$, et al. Clinical outcome of mild fetal ventriculomegaly. Am J Obstet Gynecol 1998;178:218-22.

11 Bloom SL, Bloom DD, DellaNebbia C, et al. The developmental outcome of children with mild isolated ventriculomegaly. Obstet Gynecol 1997;90:93-7.

12 Graham E, Duhl A, Ural S, et al. The degree of antenatal ventriculomegaly is related to pediatric neurological morbidity. J Matern Fetal Med. 2001: 10;258-63).

13 Patel MD, Filly AL, Hersch DR, et al. Isolated mild fetal cerebral ventriculomegaly: clinical course and outcome. Radiology 1994;192:759-64.

14 Gupta JK, Bryce FC, Lilford RJ. Management of apparently isolated fetal ventriculomegaly. Obstet Gynecol Surv 1994;49:716-21.

15 Bannister CM, Russell SA, Rimmer S, et al. Pre-natal ventriculomegaly and hydrocephalus. Neurol Res 2000;22:37-42.

16 Levene MI. Measurement of the growth of the lateral ventricles in preterm infants with real-time ultrasound. Arch Dis Child 1981;56:900-4.

17 Arora A, Bannister CM, Russell S, et al. Outcome and clinical course of prenatally diagnosed cerebral ventriculomegaly. Eur J Pediatr Surg 1998;8(suppl 1):63-4. 
18 Nicolaides $\mathbf{K H}$, Berry S, Snijders RJM, et al. Fetal lateral cerebral ventriculomegaly: associated malformations and chromosomal defects. Fetal Diagn Ther 1990;5:5-14.

19 Goldstein RB, La Pidus AS, Filly RA, et al. Mild lateral ventricular dilatation in utero: clinical significance and prognosis. Radiology 1990;176:237-42.

20 Reiss AL, Abrams MT, Greenlaw R, et al. Neurodevelopmental effects of the FMR-1 full mutations in humans. Nat Med, 1995;1;159-67.

21 Beke A, Csabay L, Rigo J, et al. Follow up studies of newborn babies with congenital ventriculomegaly. J Perinat Med 1999;27:495-505.

22 Greco P, Vimercati A, De Cosmo L, et al. Mild ventriculomegaly as a counselling challenge. Fetal Diagn Ther $2001 ; 16: 398-401$.

23 Dommergues M, Mahieu-Caputo D, Fallet-Bianco C, et al. Fetal serum interferon-alpha suggests viral infection as the aetiology of unexplained lateral cerebral ventriculomegaly. Prenat Diagn 1996;16:883-92.

24 Kelly EN, Allen VM, Seaward G, et al. Mild ventriculomegaly in the fetus, natural history, associated findings and outcome of isolated mild ventriculomegaly: a literature review. Prenat Diagn 2001;21:697-700.
25 Amato $M$, Huppi $P$, Durig $P$, et al. Fetal ventriculomegaly due to isolated brain malformations. 1990;21:130-2.

26 Bayley N. Bayley scales of infant development, 2nd ed. San Antonio, TX: Harcourt Brace \& Co, 1993

27 Milani-Comparetti A Gidoni EA. Routine developmental examination in the newborn and retarded children. Dev Med Child Neurol 1967;9:631-6

28 Senat MV, Bernard JP, Schwärzler $P$, et al. Prenatal diagnosis and follow-up of 14 cases of unilateral ventriculomegaly. Ultrasound Obstet Gynecol 1999:14:327-32.

29 Gilmore JH, van Tol J, Streicher HL, et al. Outcome in children with mild fetal ventriculomegaly: a case series. Schizophr Res 2001;48:219-26.

30 Griffiths $\mathbf{R}$. The abilities of babies Amersham: Association for Research in Infant and Child Development, 1976

31 Bellman M, Lingam S, Aukett A. Schedule of growing skills. Windsor: NFER-Nelson Publishing Company, 1996.

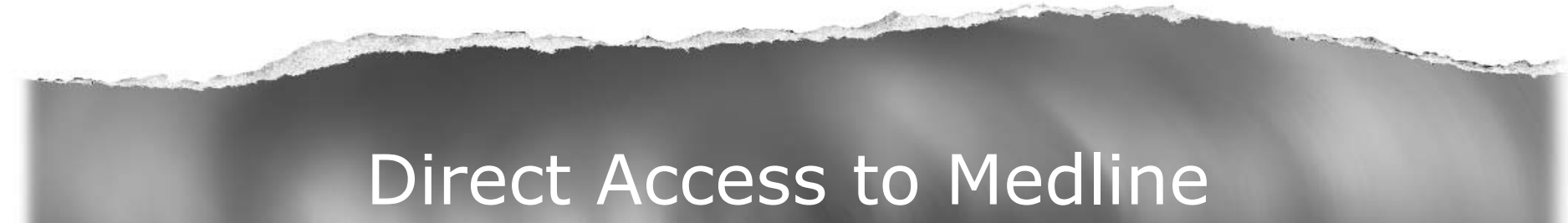

\section{Medline}

Link to Medline from the homepage and get straight into the National Library of Medicine's premier bibliographic database. Medline allows you to search across 9 million records of bibliographic citations and author abstracts from approximately 3,900 current biomedical journals.

www.archdischild.com 